\title{
Mit Werten durch die Krise? Deutsche Außenpolitik in der COVID-19 Pandemie. Ein Bericht zum Berliner Forum Außenpolitik 2020 der Körber-Stiftung
}

\author{
Liana Fix $\cdot$ Leonie Stamm
}

Eingegangen: 18. Februar 2021 / Angenommen: 3. Mai 2021 / Online publiziert: 9. Juni 2021

(C) Springer Fachmedien Wiesbaden GmbH, ein Teil von Springer Nature 2021

\section{Einleitung}

Die Coronakrise betrifft nicht nur unser tägliches Leben, sie stellt auch die Säulen der deutschen Außenpolitik in Frage: internationale Zusammenarbeit, europäische Integration und wirtschaftliche Globalisierung. Während dieser transformativen Krise fand die deutsche EU-Ratspräsidentschaft statt. Diskussionen über europäische Solidarität, Krisenbewältigung und Wiederaufbau prägten die Agenda der Ratspräsidentschaft. Gleichzeitig mussten andere dringende Themen, wie die Beziehungen zu China und den Vereinigten Staaten, angegangen werden. Das Berliner Forum Außenpolitik 2020 fand in Kooperation mit dem Auswärtigen Amt statt und stand ganz im Zeichen dieser Fragen und Debatten. Es bot eine ausgezeichnete Gelegenheit, die aktuellen Herausforderungen deutscher Außenpolitik während der COVID-19Pandemie mit internationalen und nationalen Gästen aus Politik, Regierung, Thinktanks und Medien digital zu diskutieren. Die jährliche Publikation The Berlin Pulse steuerte neue Umfrageergebnisse zu außenpolitischen Einstellungen der Deutschen zur Debatte bei.

\section{Europa in der Krise - weiterhin eine Wertegemeinschaft?}

Eröffnet wurde der Konferenztag mit einer Diskussion zwischen dem Bundesminister des Auswärtigen Heiko Maas und seinen Amtskollegen der Ratspräsidentschaft aus Portugal, Augusto Santos Silva, sowie aus Slowenien, Anže Logar. Das Trio zeigte sich vor Ort vereint im Anliegen, europäische Solidarität in der Krise zu stärken. Gleichzeitig war das Veto Polens und Ungarns in den Verhandlungen über den

Dr. Liana Fix $(\bowtie) \cdot$ Leonie Stamm

Körber-Stiftung, International Affairs, Pariser Platz 4a, 10117 Berlin, Deutschland

E-Mail: fix@koerber-stiftung.de 
Mehrjährigen Finanzrahmen sowie den Wiederaufbaufonds eine schwere Belastung für die Europäische Union. Erstmals sollte es mit dem Konditionalitätsmechanismus nämlich eine Verknüpfung von EU-Mitteln mit der Einhaltung rechtsstaatlicher Standards geben. Heiko Maas zeigte sich am 24. November optimistisch, dass internationaler Druck und Verantwortungsbewusstsein Polen und Ungarn zu einer Zustimmung bewegen werde (von der Burchard 2020a). Tatsächlich haben Polen und Ungarn ihre Blockade aufgegeben, jedoch nur nach deutlichen Zugeständnissen. Danach wurde die erfolgreiche Verabschiedung des Pakets mit einem beispiellosen Volumen von rund 1,8 Bio. $€$ als die größte Errungenschaft der deutschen Ratspräsidentschaft gefeiert. Gleichzeitig wurde auch die Verwässerung des Rechtsstaatmechanismus durch den Druck von Polen und Ungarn beklagt. Deutschland habe hier Handlungsfähigkeit über Prinzipientreue gestellt. Diese Debatte machte jedoch einen viel größeren Konflikt deutlich - die Wertedebatte innerhalb der Europäischen Union, eine Debatte um die Grundpfeiler der EU. Denn worin sich die Außenminister einig waren: Ein Grundzusammenhalt in der EU entsteht nur durch gemeinsame Werte, auf denen die EU basiert - Demokratie, Freiheit, Menschenrechte und Rechtsstaatlichkeit.

\section{3 „Grün, digitalisiert, solidarisch und feministisch“?}

Auch Pedro Sánchez Pérez-Castejón, Präsident der spanischen Regierung, der live aus Madrid zugeschaltet war, zeigte sich zuversichtlich, dass die EU ihre ambitionierten Ziele in der Krise erreichen könne (Presidencia del Gobierno 2020). Laut ihm solle sich die EU nicht nur auf den bisherigen Werten ausruhen, sondern sich weiterentwickeln: „Das kommende Europa muss grün, digitalisiert, solidarisch und feministisch sein". ${ }^{1}$ Gleichzeitig kritisierte er das Einstimmigkeitsgebot in der Europäischen Union und sprach sich für das Mehrheitsprinzip aus, um auch mit schwierigen Partnern umgehen zu können. Wie kompliziert der Umgang mit schwierigen Partnern in der EU ist, wurde in der nachfolgenden Conflict Zone deutlich (MacKenzie 2020). Sarah Kelly führte in Kooperation mit der Deutschen Welle ein Interview mit Ryszard Antoni Legutko, Mitglied des Europäischen Parlaments und Politiker der polnischen Partei Recht und Gerechtigkeit (PiS) durch. Legutko bezeichnete im Interview den Rechtsstaatmechanismus als ,eklatanten und schamlosen Verstoß gegen die EU-Abkommen“. Auf Kritik, dass Polen Pressefreiheit, Rechtsstaatlichkeit sowie die Rechte von Frauen und LGBTQ+-Personen einschränke, reagierte Legutko offensiv. Diese Kritik würde auf Fehlinformation beruhen, außerdem seien diese moralischen Belange keine Angelegenheit der EU, sondern die Sache Polens. Er sei nicht besorgt über eine Isolation Polens in der europäischen Wertegemeinschaft, sondern über die „Tyrannei der Mehrheit“ in der Europäischen Union.

\footnotetext{
1 Soweit nicht anders vermerkt, basieren die Zitate der Konferenz auf den vorliegenden Redetexten oder auf eigener Transkription und/oder Übersetzung. Mitschnitte der Panel-Debatten finden Sie auf der Internetseite des Berliner Forum Außenpolitik: https://www.koerber-stiftung.de/berliner-forum-aussenpolitik/ uebersicht-berliner-forum-aussenpolitik/forum-2020. Zugegriffen: 13. Apr. 2021.
} 


\section{Ein warmer Luftzug über dem Atlantik}

Der Nachmittag beim Berliner Forum Außenpolitik stand ganz im Zeichen der USWahlen. Auch hier zog sich die Wertediskussion wie ein roter Faden durch die Debatten. Minister des Auswärtigen Heiko Maas zeigte sich davon überzeugt, dass die transatlantische Zusammenarbeit gerade jetzt wichtiger denn je sei, insbesondere angesichts der Jahrhundertthemen Pandemiebekämpfung, Klima, Migration und Digitalisierung. Samantha Power, nominiert als künftige Chefin der US-Entwicklungsbehörde USAID, verbreitete nach vier Jahren der Unsicherheit Optimismus und bezeichnete im Gespräch mit Karen Donfried, Präsidentin des German Marshall Fund und nominiert als Assistant Secretary of State for European and Eurasian Affairs, die neue Biden-Administration als einen ,warmen Luftzug, der über den Atlantik weht“. Bidens Rückkehr werde ganz unter dem außenpolitischen Motto „America's back: you can count on us“ stattfinden. Doch um das Vertrauen wiederaufzubauen, gehöre mehr dazu. Samantha Power schlug drei Themen vor, die die USA prioritär in Angriff nehmen sollten (Power 2021): Erstens, eine Führungsrolle in der globalen Impfverteilung einzunehmen. Zweitens, die USA wieder attraktiv zu machen für internationale Studierende und akademische Exzellenz. Drittens, den Kampf gegen Korruption anzugehen. Deutschland solle diese Gelegenheit beim Schopf packen und die amerikanisch-deutsche „Beziehungskrise“ hinter sich lassen. Helfen könne hier die Rückbesinnung auf die gemeinsamen demokratischen Werte.

\section{Die Illusion strategischer Autonomie}

Die Notwendigkeit eines Angebots an die USA betonte auch die deutsche Verteidigungsministerin Annegret Kramp-Karrenbauer im Interview mit Der Spiegel. Europa müsse die neue Administration durch ein politisches Entgegenkommen unterstützen. Auf die Kontroverse zwischen ihr und dem französischen Präsidenten Emmanuel Macron angesprochen (Thumann 2020), warb Kramp-Karrenbauer dafür, dass Europa sich nicht der Illusion hingeben solle, ohne die USA für seine eigene Sicherheit sorgen zu können - diese Vision einer strategischen Autonomie ginge zu weit (von der Burchard 2020b). Zuspruch erhielt die deutsche Verteidigungsministerin in diesem Punkt von Pedro Sánchez Pérez-Castejón: Er unterstütze in dieser Debatte die deutsche Interpertation. Die deutsche Bevölkerung ist hingegen gespalten. $51 \%$ sagten in der Umfrage des The Berlin Pulse im November 2020, dass Deutschland unabhängiger von den USA werden solle. Interessant ist dies insbesondere vor dem Hintergrund des Erstarkens Chinas. Neutralität (82\%) ist für die Deutschen die präferierte Antwort auf die Frage, welcher Seite sich Deutschland im Falle eines Kalten Krieges zwischen China und den USA zuwenden sollte. Die Diskrepanz zwischen der Wahrnehmung der deutschen Bevölkerung auf der einen Seite und der außen- und sicherheitspolitischen Community auf der anderen Seite wurde beim Forum sehr deutlich: Bei einer Umfrage vor Ort sagten $40 \%$ der Teilnehmenden, dass sich Deutschland im Falle eines Kalten Krieges auf die Seite der USA stellen solle, während im Vergleich nur $58 \%$ für Neutralität votierten. 


\section{Die große Entkopplung?}

„Wie umgehen mit China?“ war auch das Thema der Nachmittagsdiskussion „A Great Decoupling? The US-Europe-China Triangle“. Zugeschaltet waren StormyAnnika Mildner, damalige Abteilungsleiterin für Außenwirtschaftspolitik beim Bundesverband der Deutschen Industrie, nun Direktorin des Aspen Institute Deutschland, Joseph S. Nye Jr., ehemaliger Professor und Dekan der Harvard Kennedy School of Government, Keyu Jin, Associate Professor of Economics an der London School of Economics and Political Science, und Kishore Mahbubani, Distinguished Fellow am Asien-Forschungsinstitut der National University of Singapore. Insbesondere durch die Pandemie seien die bereits existierenden geopolitischen Spannungen massiv verschärft worden. Wer sich nun durch den Ausgang der US-Wahl jedoch in Sicherheit wiege, liege falsch: Laut Mildner seien diese Spannungen viel mehr als ein personifizierter Konflikt zwischen dem damaligen US-Präsidenten Trump und der chinesischen Führung, sondern ein Konflikt der Werte und ein Wettbewerb der Einflusssphären. Die Expert*innen waren sich einig, dass eine umfassende Entkopplung zwischen China und den USA nicht zu begrüßen sei. Gleichzeitig darf Europa nicht zum Spielball werden im Großmächtewettbewerb zwischen den USA, Russland und China, sondern müsse eine aktivere Rolle einnehmen. Das bekräftigte auch Kishore Mahbubani. Das 21. Jahrhundert hätte andere Strukturen; Entscheidungen würden nicht mehr nur in London, Paris, Berlin getroffen. Europa müsse jedoch, gerade gegenüber China, Grenzen aufzeigen und mit weiteren asiatischen Ländern zusammenarbeiten, um ein Gegengewicht zu bilden.

\section{Gemeinsame Werte auch im digitalen Raum}

Eric Schmidt, Vorsitzender der Nationalen Sicherheitskommission für Künstliche Intelligenz der USA und ehemaliger Executive Chairman und CEO bei Google, betonte in einer Diskussion in Kooperation mit dem Aspen Institute Deutschland, dass gemeinsame Werte und Normen auch im digitalen Raum die größte Stärke der USA und Europas seien: „Wir sind stärker im Kollektiv, wenn wir gemeinsam handeln.“ Er betonte jedoch, dass es nicht die oft gewünschte Global Digital Order geben würde: „Es hängt von dem eigenen Model ab, wie eine digitale Ordnung erreicht wird." China sei durch Digitalisierung autoritärer geworden, die USA diffuser. Anstatt eine globale Ordnung anzuvisieren, sollten Europa und die USA das „Gezänk“ einstellen, und Systeme aufbauen, die demokratische Werte reflektieren. Dazu sei harte Arbeit notwendig, um diese zu implementieren - insbesondere angesichts der Konkurrenz, die unter einem vollkommen anderen Wertesystem agiere.

\section{Vernetzung in Zeiten der Krise}

Internationale Verständigung muss in Zeiten der Krise neue digitale Wegen finden. Das Berliner Forum Außenpolitik 2020 setzte sich zum Ziel, die Krise als Chance zu nutzen und Dialog und Austausch zu den außenpolitischen Kernthemen 
für Deutschland und Europa einem noch breiteren Publikum zugänglich zu machen. Mehr als 1000 geladene Gäste schalteten sich digital über eine Konferenzplattform zum Berliner Forum Außenpolitik zu. Die Vernetzung der Teilnehmenden untereinander wurde außerdem durch Breakout Sessions in kleinem Rahmen ermöglicht, unter anderem mit der ehemaligen schwedischen Außenministerin Margot Wallström zu feministischer Außenpolitik sowie mit dem ehemaligen australischen Premierminister Kevin Rudd zu Multilateralismus. Neben den geladenen Teilnehmenden erreichte das Forum ein breites europäisches und internationales Publikum mit über 70.000 Livestream-Zuschauer*innen. Auch in Pandemiezeiten wurde so das ,Miteinander statt übereinander reden“ ermöglicht und ein Forum für außenpolitische Debatten geschaffen.

\section{Literatur}

von der Burchard, H. (2020a, 24. Nov.). Heiko Maas optimistic on EU breaking budget deadlock. Politico. https://www.politico.eu/article/german-foreign-minister-optimistic-budget-deadlock-can-beresolved-in-the-coming-days/?utm_source=POLITICO.EU\&utm_campaign=bde0a606c1-EMAIL_ CAMPAIGN_2020_11_25_06_10\&utm_medium=email\&utm_term=0_10959edeb5-bde0a606c1190427305. Zugegriffen: 28. Jan. 2021.

von der Burchard, H. (2020b, 24. Nov.). German defense minister expresses surprise over Macron criticism. Politico. https://www.politico.eu/article/annegret-kramp-karrenbauer-defense-germany-natomacron-alliance. Zugegriffen: 28. Jan. 2021.

MacKenzie, A. (2020, 26. Nov.). Can the Polish government afford to be at odds with the EU? Deutsche Welle. https://www.dw.com/en/can-the-polish-government-afford-to-be-at-odds-with-the-eu/ a-55740424. Zugegriffen: 28. Jan. 2021.

Power, S. (2021). The can-do-power. America's advantage and Biden's chance. Foreign Affairs. https:// www.foreignaffairs.com/articles/united-states/2020-11-20/samantha-power-can-do-power. Zugegriffen: 28. Jan. 2021.

Presidencia del Gobierno (2020). Pedro Sánchez stresses that European Union will come out of crisis stronger. https://www.lamoncloa.gob.es/lang/en/presidente/news/Paginas/2020/20201124korberforum.aspx. Zugegriffen: 28. Jan. 2021.

Thumann, M. (2020, 26. Nov.). Die EU muss ihre Position zu den USA neu vermessen. Zeit Online. https://www.zeit.de/politik/ausland/2020-11/strategische-autonomie-europa-verteidigungemmanuel-macron-annegret-kramp-karrenbauer. Zugegriffen: 28. Jan. 2021. 